\title{
Open X Laparoscopic X Robotic Simple Prostatectomy: A Comparative Study among Surgeons at Different Stages of Learning Curve
}

\author{
Tobias-Machado $\mathbf{M}^{* 1,2}$, $\mathrm{x}$ Silva Cunha $\mathrm{FT}^{1}$, Pazeto $\mathrm{CL}^{1}$ and Luz $\mathbf{M}^{2}$ \\ ${ }^{1}$ Department of Urology, Faculdade de Medicina do ABC, São Paulo, Brazil \\ ${ }^{2}$ Hospital São Luís Rede D’or, São Paulo, Brazil
}

*Corresponding author: Marcos Tobias-Machado, Section of Minimally invasive surgery, Department of Urology, Faculdade de Medicina do ABC, SP, Brazil, Tel: +5511999329944; Email: tobias-machado@uol.com.br

\section{Research Article}

Volume 5 Issue 1

Received Date: April 08, 2020

Published Date: April 21, 2020

DOI: $10.23880 /$ oajun-16000176

\section{Abstract}

Introduction and Objective: Laparoscopic and robot-assisted simple prostatectomies are considered feasible options for treatment of benign prostate hyperplasia (BPH). We sought to compare perioperative and functional outcomes during learning curve of surgeons for open, laparoscopic and robotic techniques.

Material and Methods: Data was prospectively collected from 100 patients subjected to simple prostatectomy. Demographic and perioperative data were evaluated. Postoperative success, measured by improvement in IPSS and Qmax, and patient satisfaction were compared. ANOVA test was used to assess quantitative data and compare means. Chi-square test was used to compare categorical variables.

Results: Sixty patients underwent Open Simple Prostatectomy (OSP), 20 Laparoscopic Simple Prostatectomy (LSP) and 20 Robot assisted technique (RASP). Mean catheter time (OSP: 8,5 x LSP: 5 x RASP: 4 days, p=0,04) and hospital stay (OSP: 4,5 x LSP: 2, 2 RASP: 2 days, $p=0,04$ ) were higher on patients submitted to open approach. There was no statistically difference in irrigation time (OSP: 2,7 x LSP: 1,5 x RASP: 1,5 days, $\mathrm{p}=0,06$ ). Intraoperative bleeding (OSP: $1000 \mathrm{x}$ LSP: $530 \mathrm{x}$ RASP: $350 \mathrm{ml}, \mathrm{p}=0,02$ ) and need for transfusion (OSP: 5/60 x LSP: 1/20 x RASP: 0/20 cases, $\mathrm{p}=0,003$ ) were higher in OSP group. No evidence of statistically difference of IPSS or Qmax improvements and patient satisfaction between-groups were found. Conclusion: Simple prostatectomy can be safely and effectively performed in a minimally invasive fashion for surgeons at learning curve. Significant gains were observed in reduction of severe complication, blood loss, need for transfusion and hospital stay compared to open surgery.

Keywords: Prostatic Hyperplasia; Prostatectomy; Morbidity; Learning Curve

Abbreviations: BPH: Benign Prostatic Hyperplasia; LSP: Laparoscopic Simple Prostatectomy; OSP: Open Simple Prostatectomy; RASP: Robot assisted technique; MISP: Minimally Invasive options for Simple Prostatectomy.

\section{Introduction}

Bladder outlet obstruction in association with Benign
Prostatic Hyperplasia (BPH) represents one of the most common causes for lower urinary tract symptoms in men [1]. It is estimated that 1.1 billion individuals will be affected by this condition in 2018 [2]. The surgical treatment for BPH was first described as its standard open technique more than a hundred years ago. In early 1900's Peter Fryer [3] published the case report of the first trans vesical prostatectomy ever 


\section{Open Access Journal of Urology \& Nephrology}

to be performed. Forty seven years later, Terrence Millin developed a trans capsular technique equally effective and remarkable4. For decades, the open adenomectomy represented the standard surgical technique for severe bladder outlet obstructions associated with prostate larger than $80 \mathrm{gr}[4,5]$. Since then, medical therapy has refined the surgical indications and progressive advance in both surgical techniques and anesthetic procedures have been implicated in a decrease of surgical complications, improving long term surgical outcomes. Nevertheless, the morbidity inherent to open surgical procedures has led to the development of minimally invasive therapies to BPH [6].

According to the current guidelines, surgery is the standard procedure to most of the symptomatic cases of BPH after drug therapy failure. The decision making among one of the various techniques must consider the prostate volume. Transurethral surgery and its variations are traditionally indicated to men with a prostate volume lower than $80 \mathrm{cc}$. For bigger adenomas, with a size greater than $80 \mathrm{cc}$, simple prostatectomy is the standard procedure, especially in centers where laser enucleation is not available.

During the last decade, studies have shown that the minimally invasive simple prostatectomy, whether robot assisted or not, represents a viable alternative to treat those men with high prostate volume [7-10]. Since first series of cases published in 2002 [11], Laparoscopic Simple Prostatectomy was introduced as a potential minimally invasive alternative to surgical treatment of BPH. More recently, the Robot-assisted Laparoscopic Simple Prostatectomy is being performed replacing laparoscopic surgery when robotic system is available [12]. In a Europeanamerican multi-institutional data basis analysis, Autorino, et al. [13] claims that LSP and RALSP can both be used safely and effectively on various institutions as long as there is surgical expertise.

As open surgery is an established technique described much years ago there are few series describing recent results. As lap or robotic techniques are relatively new options, there are few data reporting results obtained during the learning curve for these procedures. This study aims to compare perioperative and functional outcomes based in results obtained during learning curve of surgeons for open, laparoscopic and robotic techniques in a contemporary series.

\section{Methods}

\section{Inclusion and Exclusion Criteria}

We performed a retrospective analysis of prospectively collected data at 3 hospitals between 2012 and 2019. Patients with prostate larger than $80 \mathrm{~g}$ and surgical. Indication such as refractory urinary retention, obstructive renal failure, failure to clinical treatment were included. Patient's preference for surgery was also taken into account. All patients had a cardiologic evaluation of risk. The study was developed in accordance with the principles of the Declaration of Helsinki and was approved by the Clinical Research Ethics Committee and the Research Commission of the three hospitals, as well as patient's informed consent. ASA 3 and 4 were excluded as well as patients with prostate cancer, neurogenic bladder disorders or urethral stenosis.

\section{Surgical Techniques}

There was no randomization in this sample. Technique was utilized based on the responsible surgeon`s choice.

Techniques for Open Simple Prostatectomy: Patients undergoing open surgery were randomly assigned to have transvesical or retropubic approach operated by a first year resident of urology tutored by one experient assistant. The techniques were standardized according to the CampbellWalsh $10^{\text {th }}$ edition [14]. In retropubic technique, a lower midline incision from umbilicus to pubic symphysis was made. Transversalis fascia was incised sharply to expose the space of Retzius. Peritoneum was mobilized cephalad and the prostate bladder junction is exposed. To achieve complete control of dorsal vein complex a 3-0 Monocryl suture was passed in the avascular plane between the urethra and dorsal vein complex at the apex of the prostate and tied and a 2-0 chromic was used to place a figure-of-eight suture deep into prostatovesical junction, to secure control of the lateral pedicles. A transverse capsulotomy in prostate 1.5 to $2.0 \mathrm{~cm}$ distal to bladder neck was performed with a scalpel. Adenoma was then bluntly dissected with Metzenbaum scissors and digitally.

When transvesical approach was chosen, anterior bladder wall was identified, and a vertical cystotomy was made with an electrocautery, right after two 3-0 Vicryl stitches were placed on each side of the midline below the peritoneal reflection. Cystotomy was then extended cephalad and caudally and a figure-of-eight suture using 3-0 Vicryl was placed and tied at the most caudal position of the cystotomy to prevent further extension of the cystotomy. With the bladder neck and prostate gland exposed, an electrocautery was used to create a circular incision in bladder mucosa distal to trigone without injuring the ureteric orifices. The plane between the prostatic adenoma and prostate pseudocapsule was bluntly dissected and prostatic adenoma was released circumferentially and inferiorly toward the apex. At the apex, prostatic urethra was transected using a pinch action of two fingertips. 


\section{Open Access Journal of Urology \& Nephrology}

After resection of the prostatic adenoma, prostatic fossa was examined and hemostatic stitches were used when needed. In addition, a 0 -chromic suture was used to place two figure-of-eight sutures to advance the bladder mucosa into prostatic fossa at 5-o'clock and 7-o'clock positions at prostatovesical junction to ensure control of main arterial blood supply to the prostate. A 22-Fr three-way Foley catheter with a $30-\mathrm{mL}$ balloon was then inserted through the anterior urethra and prostatic fossa into the bladder. In the suprapubic approach, with urethral catheter in place, prostatic pseudocapsule was closed using 2-0 absorbable sutures. In transvesical approach, cystotomy incision was closed in two layers using 2-0 absorbable sutures. Bladder was then irrigated with saline solution. A penrose drain was placed via a separate stab incision to prevent hematoma and urinoma formation.

Technique for Laparoscopic Surgery: Laparoscopic surgery was performed by last year resident of urology tutored by one experienced assistant. At this study, we performed Laparoscopic simple prostatectomy with vascular control as described by Mariano, et al. [11]. Patient was placed in a steep Trendelenburg position and 5 intraperitoneal trocars were placed in $\mathrm{W}$ fashion. All dissections were performed with a harmonic scalpel. Retzius space was dissected and preprostatic fat was removed. Endopelvic fascia was amply opened on lateral sides of the prostate. Two hemostatic sutures of 2-zero polyglactin were placed in prostatic dorsal veins and puboprostatic ligaments, and 2 in lateral pedicles of the prostate near bladder neck. Prostatic capsule and bladder neck were then opened in the midline, adenoma was dissected with a harmonic scalpel and through blunt dissection. Adenoma was removed and left in retrocecal recess. Bladder neck hemostasis was carried out with 3zero polyglactin sutures at the 5 and 7 o'clock positions, and bladder neck mucosa was advanced to prostatic fossa. A running suture of 2-zero polyglactin was used to close the capsule and bladder neck. A 22-Fr Foley catheter was introduced; the bladder was irrigated with saline solution. Adenoma was morcellated and extracted. A Penrose drain was positioned at Retzius space.

Technique for Robotic Surgery: Robotic surgery was performed by an experienced laparoscopic surgeon proctored by experienced robotic surgeon. First we gained access to Retzius space and perform Gregoir hemostatic sutures. A longitudinal incision over anterior bladder wall and prostatic capsule was managed to expose the adenoma. Two transcutaneous suprapubic sutures were placed to retract lateral bladder walls, optimizing the surgical field. For maximum exposition, posterior bladder retraction was guaranteed with Prograsp $4^{\text {th }}$ robotic arm. In sequence, mucosal incision around bladder neck was performed until the dissection plane of prostatic enucleation. Adenoma enucleation is then accomplished with monopolar robotic scissors and traction of tenaculum with pneumoperitoneum of $20 \mathrm{mmHg}$. Retrigonization of mucosa and haemostatic sutures of bladder neck was performed with intracorporeal sutures. Further hemostasis of prostatic cavity was guaranteed with bipolar and/or haemostatic agent with pneumoperitoneum of $5-10 \mathrm{mmHg}$. Finally, bladder wall was sutured in 2 plains and irrigation with saline solution through 22-Fr Owen catheter. A Vacuum drain was located at Retzius space.

\section{Data Collected}

Demographic data as age, comorbidities, preoperative IPSS and Qmax, BMI and prostate volume were obtained to evaluate if sample was balanced. Perioperative data such as surgical time, need for blood cell transfusion, complications according to Clavien-Dindo Classification, hospital stay and catheter time were evaluated. Postoperative success, measured by improvement in IPSS and Qmax, and patient satisfaction were compared.

\section{Statistical Analysis}

ANOVA test was used to assess quantitative data and compare means. Chi-square test was used to compare categorical variables. Significance level was defined as $0.05(5 \%)$. All confidence intervals used in this study were constructed with a 95\% confidence level. We used SPSS software (IBM $®$ SPSS ${ }$ Statistics 20; SPSS, Inc., Chicago, IL, USA) for calculation.

\section{Results}

One hundred patients undergoing prostate adenomectomy were included in our study. Among these patients, 60 underwent Open Simple Prostatectomy (OSP), 20 Laparoscopic Simple Prostatectomy (LSP) and 20 Robot assisted technique (RASP). Patients presented with a baseline median IPSS of 21 (range: 16-29) and a median Qmax of $7 \mathrm{ml} / \mathrm{s}$ (range: 5-11). Median prostate volume was $112 \mathrm{ml}$ (range: 81-145). There were no statistical differences on age, BMI, prostatic volume, preoperative IPSS and Qmax between-groups. Mean operative time was 125 x 180 x 150 minutes (OSP x LSP x RASP). Mean catheter time (OSP: 8.5 $x$ LSP: 5 x RASP: 4 days, $\mathrm{p}=0.04$ ) and mean hospital stay (OSP: 4.5 x LSP: 2.2 x RASP: 2 days, $p=0.04$ ) were higher on patients submitted to open approach. There was no statistically difference in irrigation time (OSP: 2.7 x LSP: $1.5 \mathrm{x}$ RASP: 1.5 days, $p=0.06$ ). Intraoperative bleeding (OSP: 1000 $x$ LSP: 530 x RASP: $350 \mathrm{ml}, \mathrm{p}=0,02$ ) and need for transfusion (OSP: $5 / 60 \times$ LSP: $1 / 20 \times$ RASP: $0 / 20$ cases, $p=0,003$ ) were higher at OSP group of patients. Occurrence of significant clot retention leading to surgical intervention (Clavien-Dindo IIIb complication) was observed on 4 cases ( $4 \%$ of all sample), 


\section{Open Access Journal of Urology \& Nephrology}

all from OSP group (6.7\%). There was no record of severe complications among LSP and RASP groups.

There was no evidence of statistically relevant difference at improvement of mean IPSS (OSP: 9 x LSP: 9 x RASP: 10, $\mathrm{p}=0.6$ ), mean Qmax (OSP:16 x LSP :15 x RASP: 16, $\mathrm{p}=0.5$ ) and patient satisfaction (OSP: $86 \%$, LSP: $85 \%$, RASP: $90 \%$, $\mathrm{p}=0.2$ ) between-groups.

\section{Discussion}

Current guidelines still indicate OSP as an effective and durable procedure for treatment of BPH and lower urinary tract symptoms among men with large prostate volume, equal or higher than $80 \mathrm{ml}$. However OSP is an invasive procedure with high morbidity and short-term postoperative rates as high as $37.1 \%$. Thus, alternative minimally invasive options for simple prostatectomy (MISP) have been introduced and implemented with the aim of reducing surgical morbidity. Endoscopic Prostatic Laser Enucleation has appeared as a good minimally invasive option to patients with larger adenomas, with excellent results when performed by trained surgeons. In our institutions prostate laser enucleation is not available, and surgeons sought to develop and gain expertise in laparoscopic approach for treatment of larger prostates. In this paper we present our experience with 90 patients surgically treated for BPH comparing outcomes of OSP, LSP and RASP. As data comparing open and minimally invasive techniques during learning curve are lacking, we assume that our study may be valuable to medical community.

Since considering our small sample, our data corroborate the findings of a series of large studies, claiming that compared with OSP, LSP was associated with less blood loss, shorter postoperative catheterization period, and shorter hospital stay, but at the expense of a longer operative time [15]. We found in our cohort that mean blood loss at OSP was significantly bigger than in LSP or RASP. OSP had a loger catheter time than LSP, which represents 70\% longer period with bladder catheterization for the OSP group. Mean stay was over two times higher on OSP group compared to LSP group. On the other hand, operative time was significantly shorter in open cases, with a surgery mean time of $125 \mathrm{~min}$ for OSP and $180 \mathrm{~min}$ for LSP. This probably occurred because open surgery is easily performed by a learning surgeon, while laparoscopic techniques demands more experience, especially for pelvic procedures. Simple Prostatectomy is a demanding procedure when performed in a minimally invasive fashion, as it includes challenging extirpative steps (adenoma dissection) and reconstructive steps (hemostasis of the prostate bed, retrigonization, and bladder suturing). As well as with other urologic procedures, such as radical prostatectomy or pyeloplasty, addition of robotic technology in simple prostatectomy can be regarded as a helpful tool for physicians embarking on this surgical endeavor. Studies addressing learning curve of laparoscopic Simple Prostatectomy are lacking.

Recently, Lucca, et al. [16] looked at 27 observational studies with 764 patients and concluded that MISP seems to be an effective and safe treatment option. Estimated blood loss in MISP seems to be lower than that reported for OSP; situation which reflects low intraoperative transfusion rates (3.5\%), and outperform those seen in laser techniques for similar-sized glands $[17,18]$. In terms of hospital stay, MISP techniques seems to offer shorter times compared to those reported in contemporary series of OSP. Our study mirrors these findings as our data shows a mean stay of 4.5 days for OSP, 2.2 days for LSP and 2 days for RASP. On RASP group, mean surgical time was $20 \%$ lower than on LSP group (LSP $180 \mathrm{~min} x$ RASP $150 \mathrm{~min}$ ). As it comes to bleeding, minimally invasive techniques seems to be more effective and safer than OSP. The group of patients that underwent RASP had a significantly smaller mean blood loss $(350 \mathrm{ml})$ in comparison with the group that underwent OSP $(1000 \mathrm{ml})$. None of MISP patients compared to 5 of OSP group required blood transfusion, due to surgical loss or complications.

Banapour, et al. [19] systematically reviewed evidence regarding RASP outcomes and identified eight published studies, all noncomparative case series. A total of 109 RASP cases were included in their analysis. In all these series, a substantial postoperative improvement in urinary symptoms was observed, suggesting that RASP is safe and effective. A low rate of overall complications was observed, being mainly mild when it occurred on LSP and RASP group. However, about $7 \%$ of OSP group presented complications graded as Clavien-Dindo IIIb.

This is a real life study, where residents are trained for open and laparoscopic surgery during residency in public hospitals and availability of robot is limited to few surgeons working in a limited number of hospitals. We identify that limited sample, lack of cost analysis, and retrospective design are the most important limitations of our study. More studies with larger experience will be necessary until minimally invasive simple prostatectomy will be considered standard for surgical treatment of larger prostates.

\section{Conclusion}

Our findings corroborate large studies designed and developed in big centers both in Europe and America, and suggest that simple prostatectomy can be safely and effectively performed in a minimally invasive fashion for surgeons at learning curve. Significant gains were observed in reduction of severe complication, blood loss, need for transfusion and hospital stay. 


\section{Open Access Journal of Urology \& Nephrology}

\section{References}

1. McVary KT, Roehrborn CG, Avins AL, Barry MJ, Bruskewitz RC, et al. (2011) Update on AUA guideline on the management of benign prostatic hyperplasia. J Urol 185(5): 1793-1803.

2. Irwin De, Kopp ZS, Agatep B, Milsom I, Abrams P (2011) Worldwide prevalence estimates of lower urinary tract symptoms, overactive bladder, urinary incontinence and bladder outlet obstruction. BJU Int 108(7): 1132-1138.

3. Freyer PJ (1900) A new method of performing perineal prostatectomy. Br Med J 1(2047): 698-699.

4. Millin T (1947) The surgery of prostatic obstructions. Ir J Med Sci (257):185-189.

5. Serretta V, Morgia G, Fondacaro L, Curto G, Lo bianco A, et al. (2002) Open prostatectomy for benign prostatic enlargement in southern Europe in the late 1990s: a contemporary series of 1800 interventions. Urology 60(4): 623-627.

6. Varkarakis I, Kyriakakis Z, Delis A, Protogerou V, Deliveliotis C (2004) Long-term results of open transvesical prostatectomy from a contemporary series of patients. Urology 64(2): 306-310.

7. Gratzke C, Schlenker B, Seitz M, Karl A, Hermanek P, et al. (2007) Complications and early postoperative outcome after open prostatectomy in patients with benign prostatic enlargement: results of a prospective multicenter study. J Urol 177(4): 1419-1422.

8. Leslie S, De Castro Abreu AL, Chopra S, Ramos P, Park D, et al. (2014) Transvesical robotic simple prostatectomy: initial clinical experience. Eur Urol 66(2): 321-329.

9. Zhou LY, Xiao J, Chen H, Zhu YP, Sun YW, et al. (2014) Extraperitoneal laparoscopic adenomectomy for benign prostatic hyperplasia. World J Urol 27(3): 385-387.

10. Porpiglia F, Fiori C, Cavallone B, Morra I, Bertolo R, et al. (2011) Extraperitoneoscopic transcapsular adenomectomy: complications and functional results after at least 1 year of follow up. J Urol 185(5): 1668-1673.

11. Mariano MB, Graziottin TM, Tefilli MV (2002) Laparoscopic prostatectomy with vascular control for benign prostatic hyperplasia. J Urol 167(6): 2528-2529.

12. Sotelo R, Clavijo R, Carmona O, Garcia A, Banda E, et al. (2008) Robotic simple prostatectomy. J Urol 179(2): 513-515.

13. Autorino R, Zargar H, Mariano MB, Sanchez-Salas R, Sotelo RJ, et al. (2015) Perioperative outcomes of robotic and laparoscopic simple prostatectomy: a europeanamerican multi-institutional analysis. Eur Urol 68(1): 86-94.

14. Han MP, Partin AW (2012) Retropubic and suprapubic open prostatectomy. In: Wein AJ, Kavoussi LR, Partin AW, Peters CA, (Eds.) 10 $0^{\text {th }}(E d n)$, Campbell's-Walsh Urology, Philadelphia: Elsevier, pp: 2683-2688.

15. Asimakopoulos AD, Mugnier C, Hoepffner JL, Spera E, Vespasiani G, et al. (2012) The surgical treatment of a large prostatic adenoma: the laparoscopic approach-a systematic review. J Endourol 26(8): 960-967.

16. Lucca I, Shariat SF, Hofbauer SL, Klatte T (2015) Outcomes of minimally invasive simple prostatectomy for benign prostatic hyperplasia: a systematic review and meta-analysis. World J Urol 33(4): 563-570.

17. Kuntz RM, Lehrich K, Ahyai SA (2008) Holmium laser enucleation of the prostate versus open prostatectomy for prostates greater than 100 grams: 5-year follow-up results of a randomised clinical trial. Eur Urol 53(1): 160-166.

18. Humphreys M, Miller N, Handa S, Terry C, Munch L, et al. (2008) Holmium laser enucleation of the prostate-outcomes independent of prostate size? J Jrol 180(6): 2431-2435.

19. Banapour P, Patel N, Kane CJ, Cohen SA, Parsons JK (2014) Robotic-assisted simple prostatectomy: a systematic review and report of a single institution case series. Prostate Cancer Prostatic Dis 17(1): 1-5. 